\title{
Diffuse alveolar haemorrhage: factors associated with in-hospital and long-term mortality
}

\author{
N. de Prost*, A. Parrot*, C. Picard*, P.Y. Ancel ${ }^{\#}$, C. Mayaud*, \\ M. Fartoukh* and J. Cadranel*
}

ABSTRACT: Diffuse alveolar haemorrhage (DAH) is a feature of several immune and nonimmune disorders. Reported prognosis is poor, with in-hospital mortality ranging from $20 \%$ to $100 \%$. Early identification of prognostic factors may be useful in the initiation of appropriate treatment.

We retrospectively analysed the charts of all patients referred to a university hospital for DAH between 1980 and 2008. Variables associated with in-hospital and long-term mortality were determined using a logistic regression model and the Kaplan-Meier method, respectively. Immunosuppressed patients were excluded.

Overall, 97 patients were included in the study. In-hospital mortality was $24.7 \%$. Factors associated with in-hospital mortality were shock (OR 77.5, 95\% Cl 8.9-677.2), glomerular filtration rate $<60 \mathrm{~mL} \cdot \mathrm{min}^{-1}$ (OR 11.2, 95\% $\mathrm{Cl} 1.8-68.4$ ) and plasmatic lactate dehydrogenase level more than twice the normal value (OR 12.1, 95\% Cl 1.7-84.3). Mortality among discharged patients was $\mathbf{1 6 . 4 \%}$ with a median follow-up duration of 34 months. Factors associated with increased longterm mortality in univariate analysis were age over 60 yrs $(p=0.026)$, cardiovascular comorbidity $(p=0.027)$ and end-stage renal failure with dependence on haemodialysis $(p=0.026)$.

Patients with immune and nonimmune DAH had similar outcomes. Early outcome depended on nonpulmonary organ failures. Conversely, late outcome was related to age, cardiac comorbidities and the need for haemodialysis.

KEYWORDS: Adult respiratory distress syndrome, haemoptysis, interstitial lung diseases, pulmonary hypertension

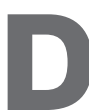
iffuse alveolar haemorrhage (DAH) is a feature of several immune and nonimmune disorders. The fundamental process common to each of the DAH syndromes is diffuse bleeding into the acinar portion of the lung. It typically presents with haemoptysis, anaemia and pulmonary infiltrates on chest radiograph. Failure to diagnose and treat DAH syndromes in their early stages may lead to acute respiratory failure and death. Reported prognosis is poor, with in-hospital mortality ranging from $20 \%$ to $100 \%$ [1-3]. Delay in initiating treatment may lead to chronic renal failure when DAH is the early manifestation of a systemic disease $[4,5]$.

Early identification of factors associated with a poor outcome may be useful in selecting the patients at highest risk to initiate appropriate treatment. To our knowledge, no publications currently address this question. Most published series are limited and instead focus on immune causes of DAH [4-6]. Long-term outcomes and factors associated with mortality in patients with DAH after discharge have not been studied. This information may be important in decision making.

Therefore, we conducted a retrospective study in a large cohort of consecutive patients hospitalised for symptomatic DAH over a $29-\mathrm{yr}$ period. Immunocompetent individuals presenting with a DAH of immune or nonimmune cause were included. We sought to identify early predictors of in-hospital mortality available within the first $24 \mathrm{~h}$ following hospital admission and factors associated with long-term mortality in discharged patients.

\section{METHODS}

\section{Study design}

A retrospective cohort study was performed in the intensive care unit (ICU) and chest department of an 800-bed tertiary hospital in France

\section{AFFILIATIONS}

* Service de Pneumologie et Réanimation and Centre de Compétence sur les Maladies Rares Pulmonaires, and

*Département de Santé Publique, Hôpital Tenon, Assistance PubliqueHôpitaux de Paris and Faculté de Médecine Pierre et Marie Curie, Université Paris VI, Paris, France.

\section{CORRESPONDENCE}

A. Parrot

Service de Pneumologie et

Réanimation

Hôpital Tenon

4 rue de la Chine

75970 Cedex Paris 20

Paris France

E-mail: antoine.parrot@tnn.aphp.fr

Received:

May 082009

Accepted after revision:

Sept 132009

First published online:

Oct 192009 
(Hôpital Tenon, Assistance Publique-Hôpitaux de Paris, Paris, France). This observational, noninterventional analysis of medical records was approved by the institutional review board of the French Learned Society for Respiratory Medicine, Société de Pneumologie de Langue Française (Paris, France).

\section{Subjects, data collection and definitions}

The medical records of consecutive patients admitted between January 1980 and May 2008 were reviewed. For patients admitted more than once, only the first admission was considered. All adult patients with symptomatic DAH were eligible. The definition of symptomatic DAH was based on the following criteria. First, the clinical and radiological presentation was compatible with the diagnosis of DAH (haemoptysis, new pulmonary infiltrates and anaemia) [4]. Secondly, the bronchoalveolar lavage (BAL) fluid was macroscopically bloody. Alternatively, haemorrhagic and siderophagic alveolitis were evidenced on BAL cytology [7], transbronchial lung biopsy or surgical lung biopsy. Diagnosis of DAH was definite when all the above criteria were met.

Patients with immunocompromised status (HIV infection, solid or haematological malignancies, bone marrow or solid organ transplantation, immunosuppressive drug therapy, cytotoxic chemotherapy or radiotherapy, steroids at a daily dose $>20 \mathrm{mg}$ of prednisone-equivalent for $>2$ months) were excluded. Patients with haemorrhage of bronchial origin and patients receiving haemodialysis for chronic renal failure were also excluded.

Variables available during the first $24 \mathrm{~h}$ of hospitalisation included demographic data (age and sex), body mass index, alcohol use (defined by the World Health Organization as $>40 \mathrm{~g} \cdot \mathrm{day}^{-1}$ for males and $>20 \mathrm{~g} \cdot \mathrm{day}^{-1}$ for females), current or former tobacco exposure, past medical history (especially respiratory and cardiovascular diseases) and ongoing anticoagulant and/or antiaggregant treatment. Clinical variables and laboratory data related to the pulmonary disorder at admission included the following: time elapsed between the first symptoms and hospital admission, respiratory rate, haemoptysis, accessory muscles use, presence of crackles, need for invasive mechanical ventilation, arterial blood gas analysis with arterial oxygen tension $\left(\mathrm{Pa}_{2} \mathrm{O}_{2}\right)$ /inspiratory oxygen fraction $\left(\mathrm{FI}, \mathrm{O}_{2}\right)$ ratio (non-mechanically ventilated patients had arterial blood gases measured while breathing room air), chest radiograph and BAL characteristics (total cell count, formula and percentage of siderophages on Perls staining and microbiology).

Nonpulmonary clinical variables were also recorded, including weight loss $>5 \%$ of baseline body weight in the last 3 months, body temperature, blood pressure, heart rate, presence of shock, extrapulmonary symptoms (skin, bone and joint, gastrointestinal, neurological, nose-ear-throat or ocular), and haematuria or proteinuria on urinalysis reagent strip. Shock was defined by a mean systemic blood pressure $<60 \mathrm{mmHg}$ after 20-30 $\mathrm{mL} \cdot \mathrm{kg}^{-1}$ starch or $40-60 \mathrm{~mL} \cdot \mathrm{kg}^{-1}$ saline, or a pulmonary capillary wedge pressure between 12 and $20 \mathrm{mmHg}$ and the need for dopamine $>5 \mu \mathrm{g} \cdot \mathrm{kg}^{-1}$ per min or dobutamine, norepinephrine or epinephrine, whatever the infusion rate, to maintain mean blood pressure $>60 \mathrm{mmHg}[8,9]$. Laboratory data included glomerular filtration rate calculated with the
Cockroft formula, haemoglobin, blood leukocyte count, platelet count, thromboplastin time, partial thromboplastin time and lactate dehydrogenase (LDH).

The time elapsed between hospital admission and the first dose of steroids, if administered, was recorded. The severity of $\mathrm{DAH}$ was assessed from generic scores of organ dysfunction including the Logistic Organ Dysfunction (LOD) score [10] and the Simplified Acute Physiology Score (SAPS) II [11]. The need for chronic haemodialysis and pulmonary disability after discharge were recorded.

The aetiologic diagnoses of DAH were specifically defined. The American College of Rheumatology criteria were used for defining a necrotising vasculitis. A histological proof of necrotising vasculitis was required [12-15]. The American Rheumatism Association criteria were used for defining a connective tissue disease [16, 17]. The anti-glomerular basement membrane antibody disease was diagnosed when the serologic test for anti-glomerular basement membrane antibodies was positive. Alternative diagnosis was made on the presence of a linear immunofluorescent glomerular immunoglobulin deposit [18]. Clinical and radiological features suggestive of DAH related to an increased pulmonary capillary pressure [19] were an increased left atrial pressure (detected by Doppler echocardiography or right heart catheterisation, which were performed in 82 and 10 patients, respectively; see figure 1 in the online supplementary material), and respiratory improvement after forced saline diuresis $[20,21]$. The diagnosis of a barotraumatic stress capillary failure due to a negative pulmonary pressure (tracheal extubation or scuba diving) was made in a suggestive context $[22,23]$. For cancer, the presence of histological evidence was used in the diagnosis. For infection, positive microbiological or serological tests allowed for diagnosis. Clotting disorders were diagnosed by a platelet count $<150,000$ cells $\cdot \mathrm{mL}^{-1}$, a patient-tocontrol subject ratio of activated partial thromboplastin time $>1.5$, or a thromboplastin time $<60 \%$. The diagnosis of toxininduced or drug-induced disease was established when there was a compatible chronology after exposure to a known

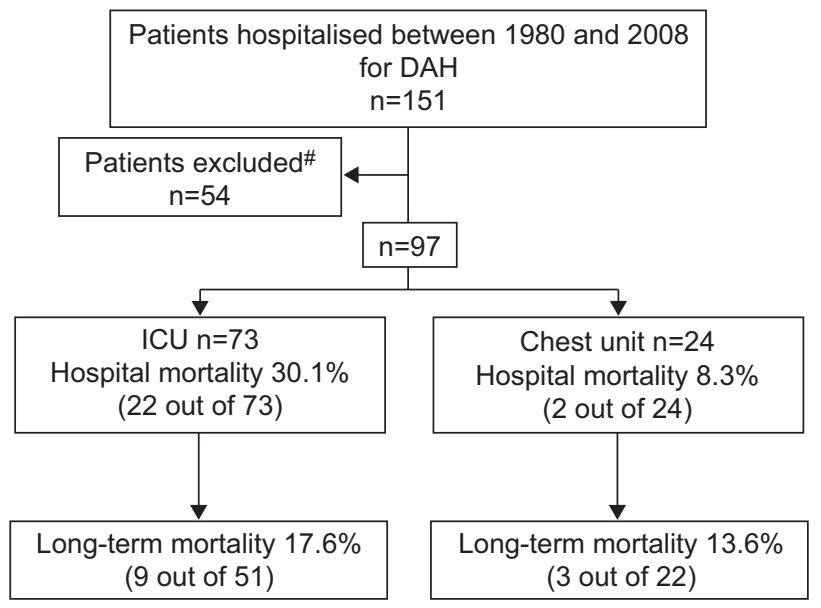

FIGURE 1. Flow chart of patients hospitalised between 1980 and 2008 for diffuse alveolar haemorrhage (DAH). ICU: intensive care unit. \#: missing data $(n=10)$, haemorrhage of bronchial origin $(n=43)$ or discovery of an immunocompromised status during hospitalisation $(n=1)$. 
pneumotoxic substance. This diagnosis required the exclusion of all the other causes of DAH [24]. Idiopathic DAH was defined when a thorough search for the above-mentioned causes remained negative.

\section{Data presentation and statistical analysis}

Standard descriptive statistics were computed. Continuous variables were reported as median (interquartile range), unless otherwise stated. The Shapiro-Wilk normality test was performed. We then drove two different models to assess the factors associated with in-hospital and long-term mortality, because we considered that each one of them addressed specific clinical issues. Univariate and multivariate logistic regressions were used to identify factors associated with inhospital mortality. Cut-off values were then defined according either to their median $\left(<60 \mathrm{~mL} \cdot \mathrm{min}^{-1}\right.$ for glomerular filtration rate) or to a clinically relevant threshold ( $>20$ pack-yrs for tobacco use, $<10 \mathrm{~g} \cdot \mathrm{dL}^{-1}$ for haemoglobin and $>2$ upper normal value for $\mathrm{LDH})$. A time variable referring to the year of hospitalisation (from 1 for 1980 to 29 for 2008) was used to assess the dependency of the outcome upon time, given the length of the study period. Variables yielding $\mathrm{p}$-values $<0.05$ by univariate analysis and considered clinically relevant were entered into a forward multivariate logistic regression analysis. Odds ratios and their 95\% confidence intervals were computed. Colinearity and interactions were tested. We chose to enter a maximum of three variables in the final multivariate model in order to avoid overfitting [25]. A base model including the main organ failures (shock and respiratory and renal failures) was created. These variables have been chosen because they have been previously shown to strongly influence the outcome of patients with acute respiratory distress syndrome [26]. The other covariates were entered in the model with a critical removal p-value of 0.1 . Effects on covariate coefficients were also considered. The SAPS II score and LOD score were not entered in the multivariate analysis because they encompassed other study variables (age as well as other variables used to define organ failures) [10, 11]. Long-term outcome was studied for patients discharged from the hospital. Factors associated with long-term mortality were identified by the Kaplan-Meier method with the log-rank test. No further multivariate analysis was performed because the statistical power was considered too low. Two-tailed p-values $<0.05$ were considered statistically significant. Analyses were carried out using Statview statistical software (SAS Institute Inc., Calasabas, CA, USA).

\section{RESULTS}

\section{Patient characteristics}

Overall, 151 patients suspected of DAH were eligible, 54 of whom were not included due to missing data $(n=10)$, haemorrhage of bronchial origin $(n=43)$ or evidence of an immunocompromised status during hospitalisation $(n=1)$ (fig. 1). Of the remaining 97 patients included in the cohort study, 73 were hospitalised in the ICU and 24 in the chest unit. The main causes of DAH are listed in table 1. One-third of the DAH syndromes were immune. The distribution of the causes of DAH over four time periods (1980-1986, 1987-1993, 19942000 and 2001-2008) is depicted in figure 2. The baseline characteristics of the 97 patients at admission are shown in table 2 .
The median length of stay was 12 (6-20) days, averaging 14 (10-22) days for ICU patients and 10 (7-15) days for conventional chest ward patients. Intravenous steroids were administered to 39 patients after a median of 2 (1-5) days after hospital admission. In-hospital mortality rate was $24.7 \%$ (24 out of 97) and did not vary significantly over time (five out of $11,45.4 \%$, during $1980-1986$; six out of $25,24 \%$, during $1987-$ 1993; five out of $29,17.2 \%$, during 1994-2000; and eight out of $32,25.0 \%$, during 2001-2008; $\mathrm{p}=0.58$ ). Accordingly, there was no relationship between the year of hospitalisation and hospital mortality in univariate analysis (OR 0.97, 95\% CI $0.91-1.03 ; \mathrm{p}=0.27$ ). The median follow-up duration was 34 (984) months for discharged patients, with a mortality rate of $16.4 \%(n=12)$. Overall mortality rate was $37.1 \%$ (36 out of 97 ).

Figure 3a shows the Kaplan-Meier curve of survival probability over time for all patients. Finally, Kaplan-Meier curves of patients having a DAH related to increased pulmonary capillary pressure or not (fig. 3b) or related to an immune cause or not (fig. 3c) were drawn for the whole study population. There was a higher overall mortality in patients with DAH related to increased pulmonary capillary pressure, as compared with patients with DAH not related to increased pulmonary capillary pressure (14 out of $26,54 \%$ versus 22 out of $71,31 \%$, respectively; $p=0.019$ by the log-rank test). In contrast, patients with immune DAH did not have a different overall prognosis, as compared with patients with nonimmune DAH (12 out of 35, 34\% versus 24 out of $62,39 \%$; $\mathrm{p}=0.738$ by the log-rank test).

\begin{tabular}{|c|c|c|}
\hline \multirow[t]{2}{*}{ TABLE 1} & \multicolumn{2}{|c|}{$\begin{array}{l}\text { Aetiology of diffuse alveolar haemorrhage (DAH) } \\
\text { syndromes }\end{array}$} \\
\hline & & Subjects $n$ \\
\hline \multicolumn{2}{|c|}{ DAH of immune cause } & 35 \\
\hline \multicolumn{2}{|c|}{ Vasculitis\# } & 25 \\
\hline \multicolumn{2}{|l|}{ AGBMAD } & 4 \\
\hline \multicolumn{2}{|c|}{ Connective tissue disease } & 6 \\
\hline \multicolumn{2}{|c|}{ DAH of nonimmune cause } & 62 \\
\hline \multicolumn{2}{|c|}{ Increased pulmonary capillary pressure } & 26 \\
\hline \multicolumn{2}{|c|}{ Systolic dysfunction of the left ventricle ${ }^{+}$} & 15 \\
\hline \multicolumn{2}{|c|}{ Diastolic dysfunction of the left ventricle } & 6 \\
\hline \multicolumn{2}{|c|}{ Valvular heart disease ${ }^{\S}$} & 5 \\
\hline \multicolumn{2}{|c|}{ Miscellaneous } & 22 \\
\hline \multicolumn{2}{|c|}{ Infection ${ }^{f}$} & 6 \\
\hline \multicolumn{2}{|c|}{ Toxic- or drug-induced DAH } & 6 \\
\hline \multicolumn{2}{|c|}{ Clotting disorder } & 4 \\
\hline \multicolumn{2}{|c|}{ Barotrauma } & 4 \\
\hline \multicolumn{2}{|c|}{ Cancer } & 2 \\
\hline \multicolumn{2}{|c|}{ Idiopathic DAH } & 14 \\
\hline \multicolumn{3}{|c|}{ 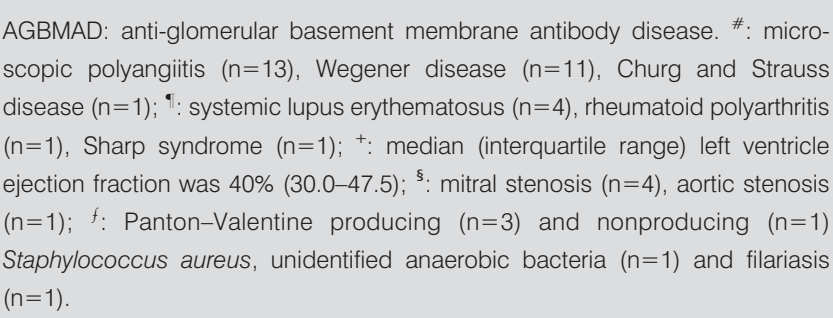 } \\
\hline
\end{tabular}




\section{Prognostic factors of hospital mortality}

24 patients $(24.7 \%)$ died in hospital, 22 of whom were hospitalised in the ICU. Age $>60$ yrs, previous cardiovascular disease, tobacco history $>20$ pack-yrs, need for invasive mechanical ventilation, shock, LDH greater than twice the normal value and glomerular filtration rate $<60 \mathrm{~mL} \cdot \mathrm{min}^{-1}$ yielded a $p$-value $<0.05$ in univariate analysis and were considered for the multivariate analysis (table 3). Three factors were independently associated with in-hospital mortality in the multivariate analysis, including shock (OR 77.6, 95\% CI 8.9-677.2), glomerular filtration rate $<60 \mathrm{~mL} \cdot \mathrm{min}^{-1}$ (OR 11.2, 95\% CI 1.8-68.4) and plasmatic LDH greater than twice the normal value (OR 12.1, 95\% CI 1.7-84.3). The Pearson goodness of fit test yielded a $p$-value $>0.05(p=0.95)$, meaning that the calibration of the model was good. The $\mathrm{r}^{2}$ yielded by the model was 0.52 .

\section{Prognostic factors of long-term outcome}

The mortality rate of the 73 discharged patients was $16.4 \%(12$ out of 73) during the follow-up period. Age $>60$ yrs $(p=0.026)$, a previous cardiovascular disease $(p=0.027)$ and a persistent renal failure requiring chronic haemodialysis $(p=0.026)$ were associated with long-term mortality in the univariate analysis performed with the Kaplan-Meier method. Figure 2 of the online supplement shows the Kaplan-Meier curves of survival representing a patient's probability of survival over time based on the presence of a previous cardiovascular disease $(p=0.027)$.

\section{DISCUSSION}

Our study reports the outcome of 97 patients hospitalised in a tertiary hospital for an initial episode of DAH. DAH may result from a broad spectrum of diseases of different pathophysiological mechanisms. However, diagnosis of the underlying condition can be challenging and may not be known within the first hours following hospital admission. We therefore aimed to identify available factors on admission that were associated with a poor prognosis. As a result, the study population reflects the large and heterogeneous spectrum of the diseases that may be encompassed in the setting of DAH. The overall mortality rate was $37.1 \%$, combining the $24.7 \%$ rate of inhospital mortality and the further post-discharge mortality rate of $16.4 \%$. Different prognostic factors were related to the inhospital and long-term mortality. The first group of factors included LDH level, shock and renal failure. The second group of factors included age $>60 \mathrm{yrs}$, cardiovascular disease and the need for chronic haemodialysis.

$\mathrm{DAH}$ is a rare and severe syndrome with a reported in-hospital mortality of $20-100 \%$ [1-3]. Previous series included limited samples of patients with immune DAH and reported that the main causes of death were infections and renal failure $[4,5,27$, 28]. Although DAH of immune causes are usually deemed to have a poorer prognosis than DAH of nonimmune causes [4], there is to our knowledge no study assessing the determinants of outcome in a cohort including DAH of immune and nonimmune causes. In our series, the in-hospital mortality rate was $24.7 \%$, emphasising the poor prognosis for patients experiencing an initial episode of DAH, even in a nonselected population of patients. These results highlight the need for a

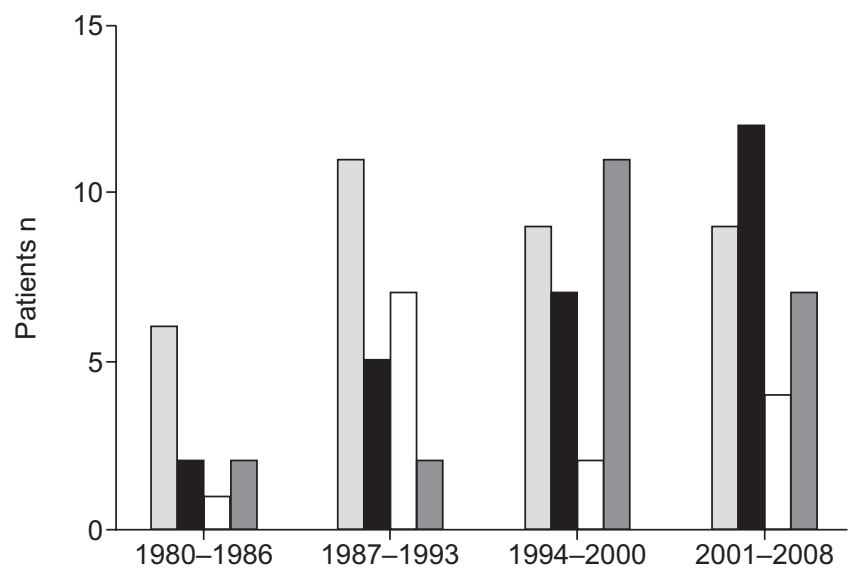

FIGURE 2. Causes of diffuse alveolar haemorrhage (DAH) for patients hospitalised over four time periods. II : immune $\mathrm{DAH}$; $\mathbf{\square}$ : increased pulmonary capillary pressure; $\square$ : idiopathic; $\square$ : miscellaneous.

tool to quickly identify patients who would benefit from aggressive treatment and intensive monitoring.

Three factors available during the first $24 \mathrm{~h}$ of hospitalisation were independently associated with in-hospital mortality. We did not enter the SAPS II and the LOD scores into our statistical model in an attempt to identify the specific organ failures related to a poor prognosis. Accordingly, the in-hospital mortality was predicted by extrapulmonary organ failures (shock and renal failure), underlining the fact that most patients were affected by general diseases (immune disorders and cardiovascular diseases) that led to cardiac and renal failure $[18,29-31]$. Half (nine out of $17 ; 53 \%$ ) of the patients with shock had a DAH related to increased pulmonary capillary pressure. Among those nine patients, eight had a pre-existing cardiovascular disease. We thus hypothesise that those patients had a cardiogenic shock associated with DAH due to increased pulmonary capillary pressure, both complicating severe/endstage left heart failure. Eight other patients, among whom only one survived, had shock: three had septic shock (three patients with Panton-Valentine producing Staphylococcus aureus) and five had a multiple organ failure syndrome of an unclear mechanism (two patients with idiopathic DAH, two patients with immune DAH and one patient with clotting disorder). However, the underlying cause of organ failure (immune versus nonimmune) was not related to outcome, as the overall mortality did not differ between patients with or without an immune cause of DAH. Surprisingly, the variables related to respiratory failure (need for invasive mechanical ventilation or $\mathrm{Pa}_{2} \mathrm{O}_{2} / \mathrm{FI}_{1} \mathrm{O}_{2}<200 \mathrm{mmHg}$ at admission) did not appear to predict mortality in our model. This may be explained by interdependence with the variable shock. Shock was indeed strongly related to hospital mortality $(p<0.0001$ for each of the respiratory failure variables). Conversely, the fact that increased $\mathrm{LDH}$ level at admission was a risk factor for hospital mortality in our series probably reflected the prognostic impact of pulmonary injury. Previous studies have demonstrated that increased LDH level in patients with pulmonary pneumocystosis or acute respiratory distress syndrome is a prognostic factor [32, 33]. However, increase in LDH level can be the result of various conditions which makes its interpretation speculative [34]. 
TABLE 2 Characteristics and initial presentation of the 97 patients

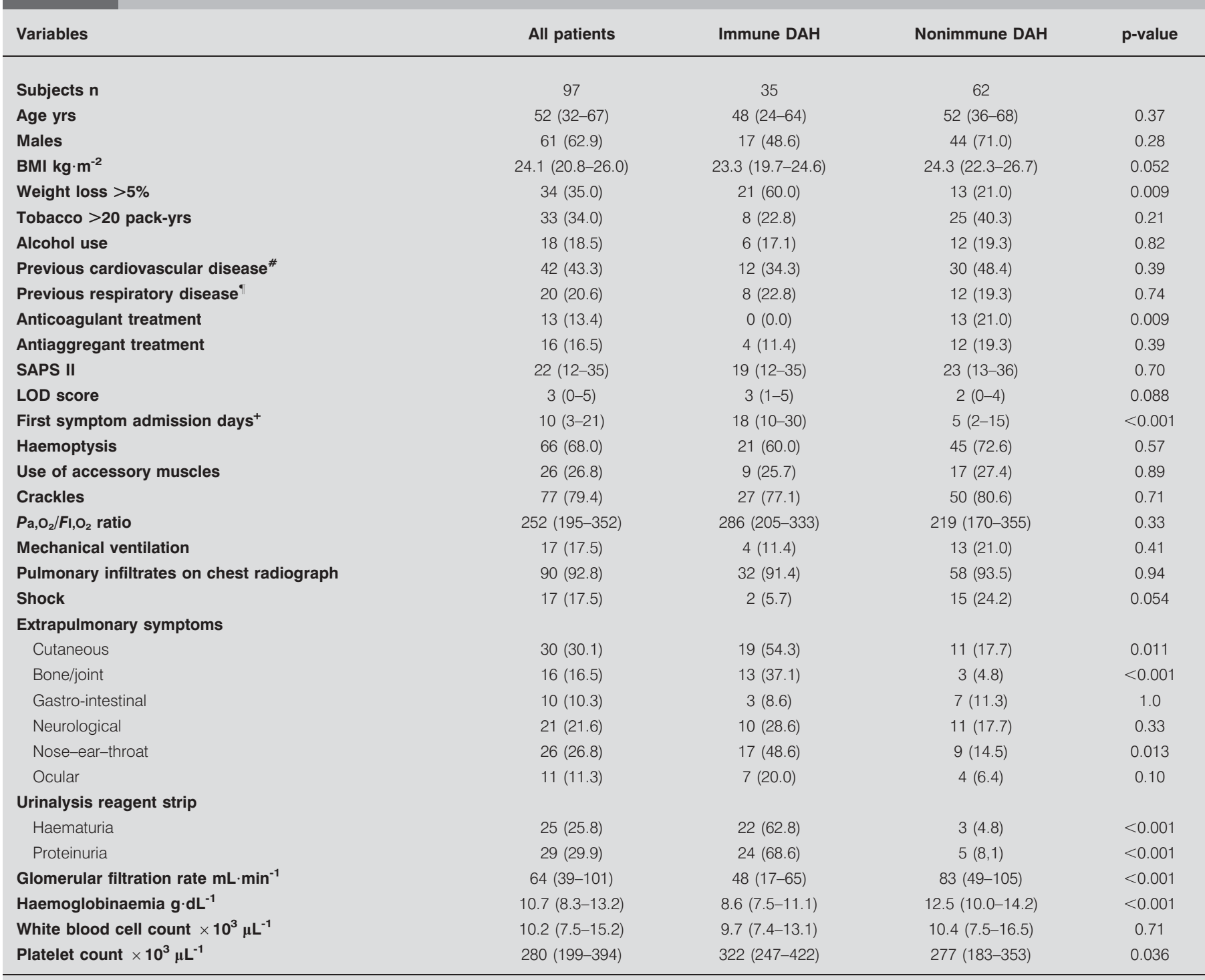

Data are presented as median (interquartile range) or $n(\%)$, unless otherwise stated. DAH: diffuse alveolar haemorrhage; BMI: body mass index; SAPS: Simplified Acute Physiology Score; LOD: Logistic Organ Dysfunction; $\mathrm{Pa}_{2} \mathrm{O}_{2}$ : arterial oxygen tension; $\mathrm{Fl}, \mathrm{O}_{2}$ : inspiratory oxygen fraction. ${ }^{\#}$ : hypertension $(\mathrm{n}=30)$, coronary heart disease $(n=15)$, atrial fibrillation $(n=11)$, oblitering arteriopathy of the lower limbs $(n=6)$, stroke $(n=3) ; "$ : chronic obstructive pulmonary disease $(n=8)$, respiratory tract infection $(n=8)$, miscellaneous $(n=4) ;{ }^{+}$: time elapsed between the first symptom of DAH and hospital admission.

12 of the 73 patients discharged from our hospital died during the follow-up period. This resulted in a low statistical power. Therefore, no multivariate analysis was performed on the factors associated with long-term outcome with the KaplanMeier method. Age $>60$ yrs was associated with mortality in discharged patients. This finding was in agreement with the study of THIBAULT et al. [35], who found that age had a greater effect on long-term mortality in a large nonselected population of patients admitted to the ICU. Dependence on haemodialysis was also a risk factor for long-term mortality. This was a result of pulmonary-renal syndrome that led to end-stage renal failure $[18,28]$. Previous studies showed that renal failure is an independent predictor of poor outcome in anti-glomerular basement membrane antibody disease [18], connective tissue disease [36] and vasculitis [37]. This suggests that a subgroup of patients suffering from immune causes of DAH leading to end-stage renal failure might be at higher risk of death, although the limited statistical power of our study did not allow us to draw such an inference. Interestingly, patients with a pre-existing cardiovascular disease had an increased risk of death after hospital discharge. This finding suggests that DAH related to increased pulmonary capillary pressure may be associated with a worse prognosis than DAH related to other causes. Most of our patients with DAH related to increased pulmonary capillary pressure (19 out of $26 ; 73.1 \%$ ) had a previously known cardiovascular disease, suggesting that $\mathrm{DAH}$ was the end-stage manifestation of a chronic heart disease that resulted in death in 12 patients. However, DAH 


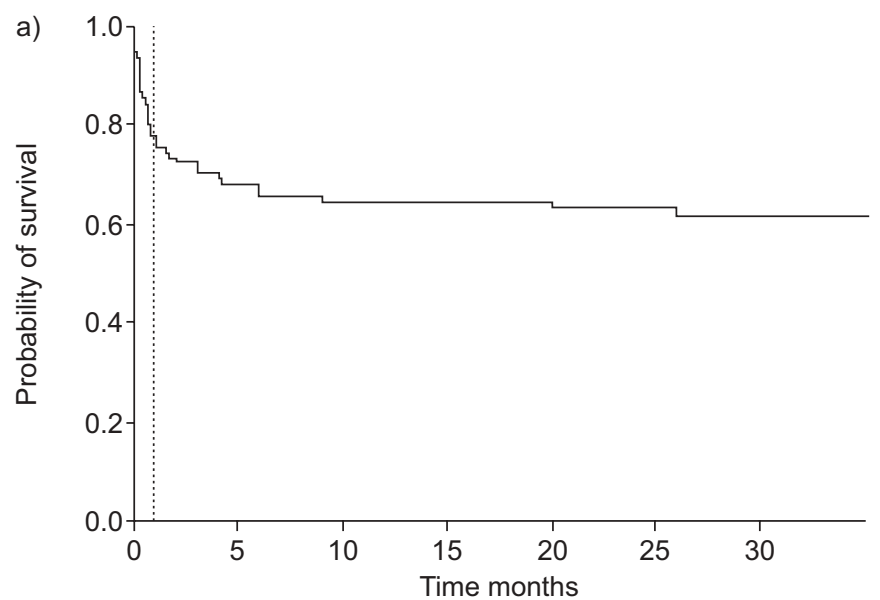

$\begin{array}{llllllll}\text { At risk } & 97 & 60 & 53 & 51 & 50 & 44 & 38\end{array}$

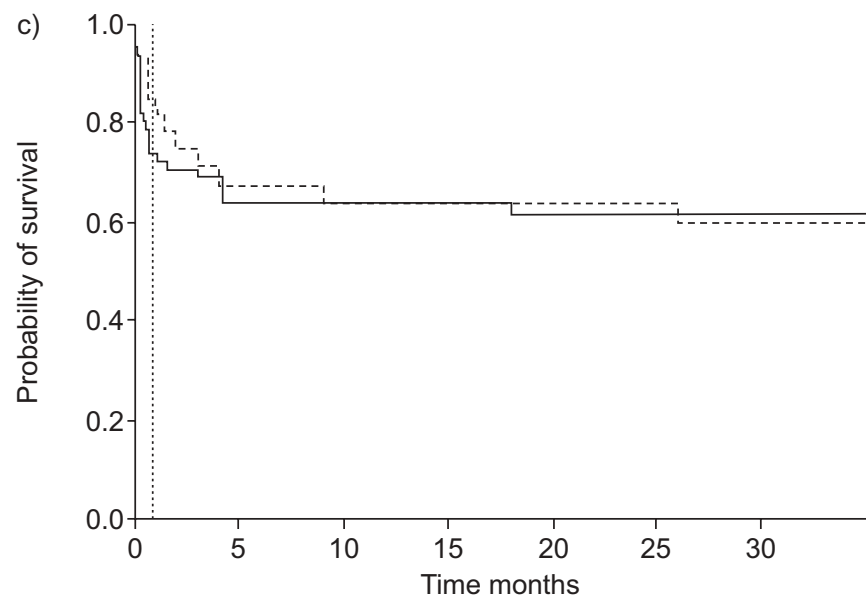

At risk

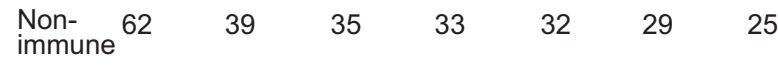

$\begin{array}{lllllll}\text { Immune } 35 & 21 & 18 & 18 & 18 & 15 & 13\end{array}$

was the first manifestation of a heart disease in seven patients (mitral stenosis, $\mathrm{n}=3$; left heart systolic dysfunction, $\mathrm{n}=3$; and diastolic dysfunction, $n=1$ ), which led to death in two. In our series, patients with DAH related to increased pulmonary capillary pressure had a $53.8 \%$ overall mortality rate. In comparison, there was a $31.0 \%$ overall mortality rate in others $(p=0.019)$. Previously published data has reported a 33\% 1-yr mortality rate in patients hospitalised for cardiogenic pulmonary oedema [29]. Our findings support this study because DAH related to increased pulmonary capillary pressure was associated with a poor prognosis. For patients hospitalised before the era of echocardiography, the pulmonary artery occlusion pressure was measured $(n=10)$. We acknowledge, however, that among patients with increased pulmonary capillary pressure, some of those hospitalised in the $1980 \mathrm{~s}$ might have been misclassified given the difficulty of assessing left ventricle diastolic function. It is thus likely that some of the patients classified as systolic dysfunction of the left ventricle had an associated diastolic dysfunction. However, all the patients classified as DAH due to increased pulmonary

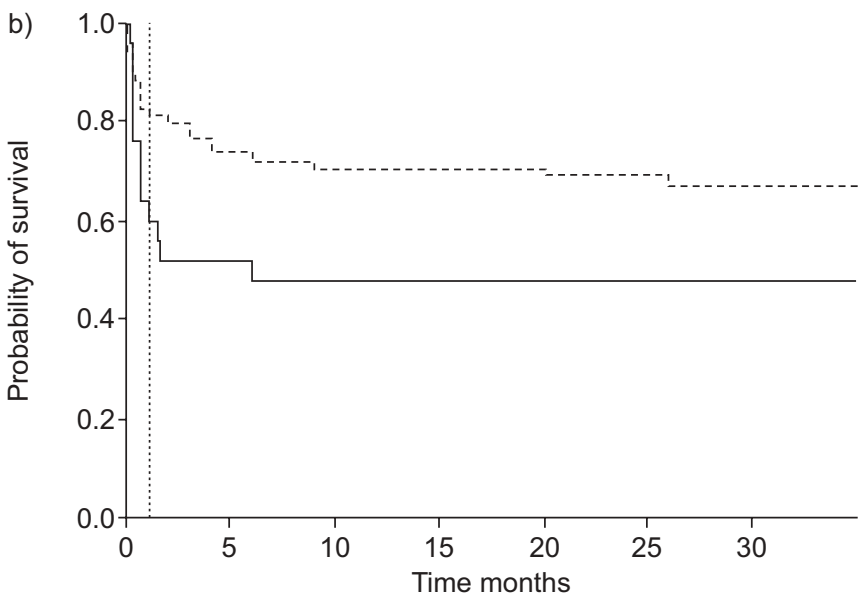

At risk

$\begin{array}{lrrrrrrr}\text { Non- } & 71 & 48 & 43 & 41 & 40 & 36 & 32 \\ \text { IPCP } & & & & & & & \\ \text { IPCP } & 26 & 12 & 10 & 10 & 10 & 8 & 6\end{array}$

FIGURE 3. Kaplan-Meier curves of the probability of survival during the median follow-up period. …... separation between in-hospital mortality and mortality in discharged patients. a) Kaplan-Meier curve showing the probability of survival for all patients. b) Kaplan-Meier curve showing the overall probability of survival for patients with $(-)$ or without $(---)$ diffuse alveolar haemorrhage $(\mathrm{DAH})$ related to increased pulmonary capillary pressure (IPCP); $p=0.019$ (log-rank test). DAH not related to IPCP includes immune and idiopathic DAH and DAH of miscellaneous causes. c) Kaplan-Meier curve showing the overall probability of survival for patients with immune $(-)$ or nonimmune $(---)$ related DAH; $\mathrm{p}=0.735$ (log-rank test). Nonimmune DAH include DAH related to IPCP, idiopathic DAH and DAH of miscellaneous causes.

capillary pressure $(n=26)$ had a patent DAH, as opposed to a usual cardiogenic alveolar oedema: BAL fluid was macroscopically bloody in 10 patients, pink in 10 and normal in six, for whom the percentage of alveolar siderophages was always $>70 \%$. The discrimination of these patients from the others was therefore challenging on hospital admission. We thus wished to include them in this series, although the mechanisms involved in these DAH are mainly related to an increase in hydrostatic pressures and not to a primary lesion of the alveolocapillary barrier, as in DAH of other causes. This subgroup of DAH seems to exhibit a worse outcome than the others and might, therefore, require a more aggressive management. Anticoagulant or antiaggregant therapy was not associated with increased in-hospital or long-term mortality in this study $(p=0.11$ and $p=0.43$, respectively). However, these treatments may exacerbate alveolar haemorrhage. In particular, this may have occurred in patients with DAH related to left heart failure. These patients frequently receive such treatments. Among the 26 patients with DAH related to increased pulmonary capillary pressure, those who received 


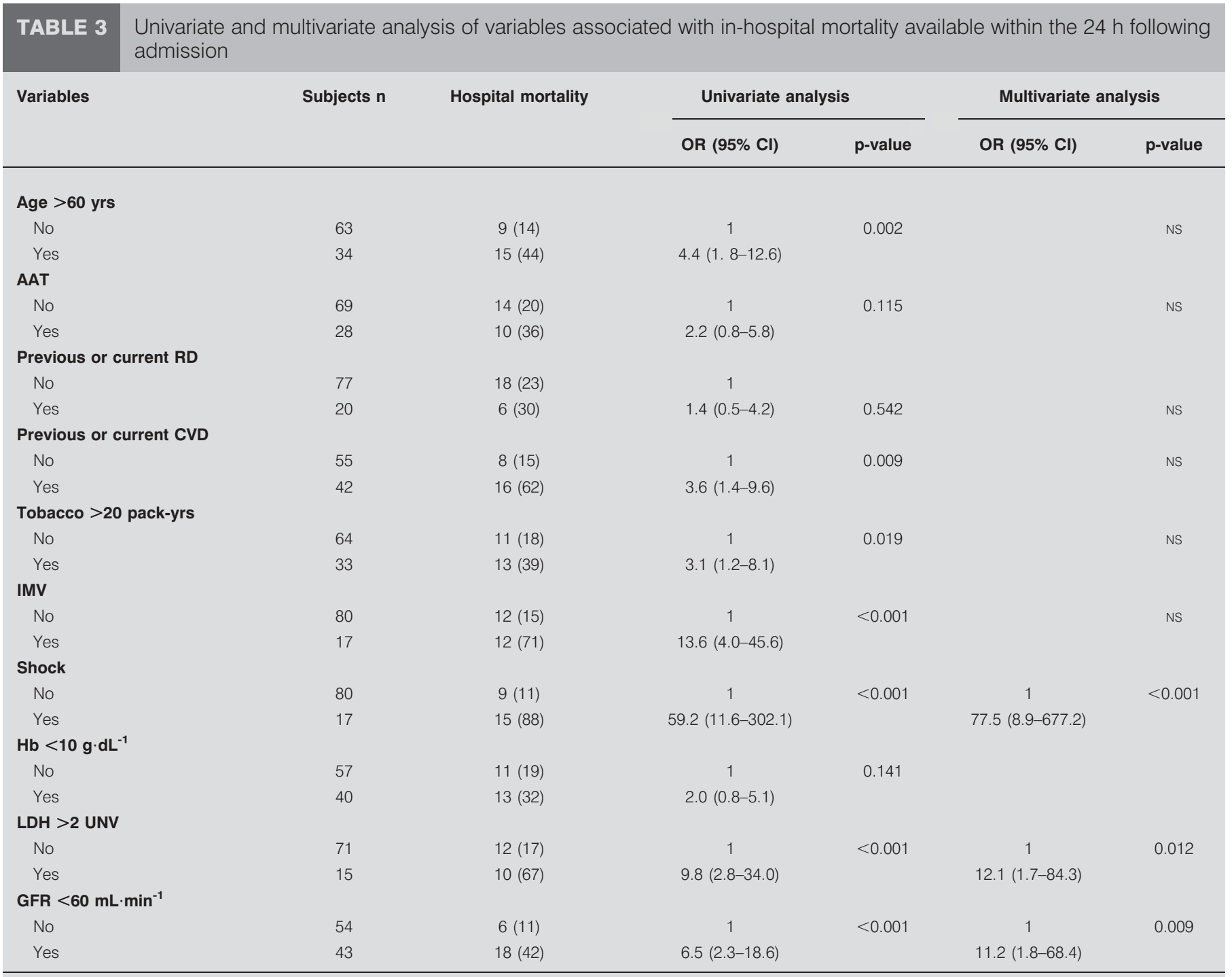

Data are presented as $n(\%)$, unless otherwise stated. AAT: anticoagulant or antiaggregant treatment; RD: respiratory disease; CVD: cardiovascular disease; IMV: invasive mechanical ventilation; $\mathrm{Hb}$ : haemoglobin; LDH: lactate dehydrogenase; UNV: upper normal value; GFR: glomerular filtration rate. The Pearson goodness of fit test showed good calibration of the model (for four degrees of freedom: Chi-squared $=0.75, p=0.94$ ).

anticoagulant or antiaggregant therapy trended towards a higher in-hospital mortality rate than the others (11 out of 18 , $61.1 \%$ versus none out of eight, $0.0 \%$; $=0.076$ ). This finding should be interpreted cautiously, as it might reflect the severity of the underlying heart disease. However, DAH has been frequently reported in patients receiving thrombolytic therapy in the setting of acute myocardial infarction. Alveolar bleeding may be facilitated by increased systolic pulmonary arterial pressure up to $65 \mathrm{mmHg}$, in accordance with animal models $[38,39]$. In rabbits, an artificial rise in capillary pressure leads to breaks in the alveolocapillary barrier [40, 41]. Such intermediate forms of pulmonary oedema, which combine increased permeability and hydrostatic pressure, have been described in patients with heart failure [42]. Whether clotting disorders may exacerbate the alveolar bleeding in the setting of capillary stress failure remains uncertain. However, withdrawing anticoagulant and/or antiaggregant therapy, at least temporarily, in patients with DAH due to increased pulmonary capillary pressure might be a suitable intervention which would need to be tested in further studies.

This study has several limitations. First, it is a retrospective study which covers a long period during which diagnosis tools, treatments and global quality of care might have varied; secondly, it is a monocentric study, which limits the potential of extrapolation of the conclusions drawn. However, the facts that 1) immunocompromised hosts were excluded; 2) the patients were all diagnosed and treated in the same institution; and 3) only basic clinical and radiological characteristics and biological tests were used to determine prognosis limit the risk of heterogeneity of practices during time. Main changes in practices over the 29 yrs of this study included 1) the increased use of Doppler echocardiography over time (from $\sim 50 \%$ in the 1980 s to up to $\sim 100 \%$ during the last 14 yrs of the study) and 
the parallel decrease in the use of right heart catheterisation (see figure 1 of the online supplement), and 2) the changes in the ventilatory management of patients with acute respiratory distress syndrome (i.e. tidal volume reduction) are likely to have improved their prognosis. Finally, the fact that a time variable was not associated with in-hospital mortality in univariate analysis does not preclude the possibility of an impact of time on outcome since the study was neither designed nor powered to test this hypothesis.

In summary, we reported a large series of immunocompetent patients hospitalised for initial episodes of DAH (immune and nonimmune). Predictors of in-hospital mortality available during the first $24 \mathrm{~h}$ following admission included shock, renal failure and increased LDH level, whatever the underlying cause of DAH. In contrast, long-term mortality was determined by age, cardiac comorbidities and dependence on haemodialysis. Further studies are needed to clarify the role of anticoagulant and antiaggregant therapy on the initial severity and outcome of DAH patients. This therapy may have a significant impact on those with left heart failure.

\section{STATEMENT OF INTEREST}

None declared.

\section{REFERENCES}

1 Benoit FL, Rulon CB, Theil GB, et al. Goodpasture's syndrome: a clinicopathologic entity. Am J Med 1964; 37: 424-444.

2 Gallagher H, Kwan JT, Jayne DR. Pulmonary renal syndrome: a 4year, single-center experience. Am J Kidney Dis 2002; 39: 42-47.

3 Zamora MR, Warner ML, Tuder R, et al. Diffuse alveolar hemorrhage and systemic lupus erythematosus. Clinical presentation, histology, survival, and outcome. Medicine (Baltimore) 1997; 76: 192-202.

4 Leatherman JW, Davies SF, Hoidal JR. Alveolar hemorrhage syndromes: diffuse microvascular lung hemorrhage in immune and idiopathic disorders. Medicine (Baltimore) 1984; 63: 343-361.

5 Travis WD, Colby TV, Lombard C, et al. A clinicopathologic study of 34 cases of diffuse pulmonary hemorrhage with lung biopsy confirmation. Am J Surg Pathol 1990; 14: 1112-1125.

6 Holguin F, Ramadan B, Gal AA, et al. Prognostic factors for hospital mortality and ICU admission in patients with ANCArelated pulmonary vasculitis. Am J Med Sci 2008; 336: 321-326.

7 De Lassence A, Fleury-Feith J, Escudier E, et al. Alveolar hemorrhage. Diagnostic criteria and results in 194 immunocompromised hosts. Am J Respir Crit Care Med 1995; 151: 157-163.

8 Annane D, Bellissant E, Cavaillon JM. Septic shock. Lancet 2005; 365: $63-78$.

9 Bone RC, Balk RA, Cerra FB, et al. Definitions for sepsis and organ failure and guidelines for the use of innovative therapies in sepsis. The ACCP/SCCM Consensus Conference Committee. American College of Chest Physicians/Society of Critical Care Medicine. Chest 1992; 101: 1644-1655.

10 Le Gall JR, Klar J, Lemeshow S, et al. The Logistic Organ Dysfunction system. A new way to assess organ dysfunction in the intensive care unit. ICU Scoring Group. JAMA 1996; 276: 802-810.

11 Le Gall JR, Lemeshow S, Saulnier F. A new Simplified Acute Physiology Score (SAPS II) based on a European/North American multicenter study. JAMA 1993; 270: 2957-2963.

12 Leavitt RY, Fauci AS, Bloch DA, et al. The American College of Rheumatology 1990 criteria for the classification of Wegener's granulomatosis. Arthritis Rheum 1990; 33: 1101-1107.
13 Lightfoot RW Jr, Michel BA, Bloch DA, et al. The American College of Rheumatology 1990 criteria for the classification of polyarteritis nodosa. Arthritis Rheum 1990; 33: 1088-1093.

14 Masi AT, Hunder GG, Lie JT, et al. The American College of Rheumatology 1990 criteria for the classification of Churg-Strauss syndrome (allergic granulomatosis and angiitis). Arthritis Rheum 1990; 33: 1094-1100.

15 Mills JA, Michel BA, Bloch DA, et al. The American College of Rheumatology 1990 criteria for the classification of HenochSchonlein purpura. Arthritis Rheum 1990; 33: 1114-1121.

16 Arnett FC, Edworthy SM, Bloch DA, et al. The American Rheumatism Association 1987 revised criteria for the classification of rheumatoid arthritis. Arthritis Rheum 1988; 31: 315-324.

17 Tan EM, Cohen AS, Fries JF, et al. The 1982 revised criteria for the classification of systemic lupus erythematosus. Arthritis Rheum 1982; 25: 1271-1277.

18 Levy JB, Turner AN, Rees AJ, et al. Long-term outcome of antiglomerular basement membrane antibody disease treated with plasma exchange and immunosuppression. Ann Intern Med 2001; 134: 1033-1042.

19 Shamsham F, Mitchell J. Essentials of the diagnosis of heart failure. Am Fam Physician 2000; 61: 1319-1328.

20 Hunt SA, Baker DW, Chin MH, et al. ACC/AHA Guidelines for the Evaluation and Management of Chronic Heart Failure in the Adult: Executive Summary. A Report of the American College of Cardiology/American Heart Association Task Force on Practice Guidelines (Committee to Revise the 1995 Guidelines for the Evaluation and Management of Heart Failure): Developed in Collaboration With the International Society for Heart and Lung Transplantation; Endorsed by the Heart Failure Society of America. Circulation 2001; 104: 2996-3007.

21 Yancy CW, Lopatin M, Stevenson LW, et al. Clinical presentation, management, and in-hospital outcomes of patients admitted with acute decompensated heart failure with preserved systolic function: a report from the Acute Decompensated Heart Failure National Registry (ADHERE) Database. J Am Coll Cardiol 2006; 47: 76-84.

22 Broccard AF, Liaudet L, Aubert JD, et al. Negative pressure posttracheal extubation alveolar hemorrhage. Anesth Analg 2001; 92: 273-275.

23 West JB, Mathieu-Costello O. Stress failure of pulmonary capillaries in the intensive care setting. Schweiz Med Wochenschr 1992; 122: 751-757.

24 Schwarz MI, Fontenot AP. Drug-induced diffuse alveolar hemorrhage syndromes and vasculitis. Clin Chest Med 2004; 25: 133-140.

25 Peduzzi P, Concato J, Kemper E, et al. A simulation study of the number of events per variable in logistic regression analysis. J Clin Epidemiol 1996; 49: 1373-1379.

26 Venet C, Guyomarc'h S, Pingat J, et al. Prognostic factors in acute respiratory distress syndrome: a retrospective multivariate analysis including prone positioning in management strategy. Intensive Care Med 2003; 29: 1435-1441.

27 Cruz BA, Ramanoelina J, Mahr A, et al. Prognosis and outcome of 26 patients with systemic necrotizing vasculitis admitted to the intensive care unit. Rheumatology (Oxford) 2003; 42: 1183-1188.

28 Hauer HA, Bajema IM, Van Houwelingen HC, et al. Determinants of outcome in ANCA-associated glomerulonephritis: a prospective clinico-histopathological analysis of 96 patients. Kidney Int 2002; 62: $1732-1742$.

29 Chatterjee K, Rame JE. Systolic heart failure: chronic and acute syndromes. Crit Care Med 2008; 36: S44-S51.

30 Leavitt RY, Fauci AS. Pulmonary vasculitis. Am Rev Respir Dis 1986; 134: 149-166.

31 Liang KV, Williams AW, Greene EL, et al. Acute decompensated heart failure and the cardiorenal syndrome. Crit Care Med 2008; 36: S75-S88.

32 Choi KW, Chau TN, Tsang O, et al. Outcomes and prognostic factors in 267 patients with severe acute respiratory syndrome in Hong Kong. Ann Intern Med 2003; 139: 715-723. 
33 Tasaka S, Hasegawa N, Kobayashi S, et al. Serum indicators for the diagnosis of pneumocystis pneumonia. Chest 2007; 131: $1173-1180$.

34 Drent M, Cobben NA, Henderson RF, et al. Usefulness of lactate dehydrogenase and its isoenzymes as indicators of lung damage or inflammation. Eur Respir J 1996; 9: 1736-1742.

35 Thibault GE, Mulley AG, Barnett GO, et al. Medical intensive care: indications, interventions, and outcomes. N Engl J Med 1980; 302: 938-942.

36 Wadee S, Tikly M, Hopley M. Causes and predictors of death in South Africans with systemic lupus erythematosus. Rheumatology (Oxford) 2007; 46: 1487-1491.

37 Booth $\mathrm{AD}$, Almond MK, Burns A, et al. Outcome of ANCAassociated renal vasculitis: a 5-year retrospective study. Am J Kidney Dis 2003; 41: 776-784.
38 Nathan PE, Torres AV, Smith AJ, et al. Spontaneous pulmonary hemorrhage following coronary thrombolysis. Chest 1992; 101: 1150-1152.

39 Yigla M, Sprecher E, Azzam Z, et al. Diffuse alveolar hemorrhage following thrombolytic therapy for acute myocardial infarction. Respiration 2000; 67: 445-448.

40 Tsukimoto K, Mathieu-Costello O, Prediletto R, et al. Ultrastructural appearances of pulmonary capillaries at high transmural pressures. J Appl Physiol 1991; 71: 573-582.

41 West JB, Mathieu-Costello O. Vulnerability of pulmonary capillaries in heart disease. Circulation 1995; 92: 622-631.

42 Sprung CL, Rackow EC, Fein IA, et al. The spectrum of pulmonary edema: differentiation of cardiogenic, intermediate, and noncardiogenic forms of pulmonary edema. Am Rev Respir Dis 1981; 124: $718-722$. 